\title{
Sine-Gordon Expansion Method for Exact Solutions to Conformable Time Fractional Equations in RLW-Class
}

\author{
Alper Korkmaz ${ }^{a, *}$ Ozlem Ersoy Hepson ${ }^{b}$, Kamyar Hosseini $^{c}$, \\ Hadi Rezazadeh ${ }^{d}$, Mostafa Eslami ${ }^{e}$ \\ ${ }^{a}$ Çankırı Karatekin University, Department of Mathematics, 18200, Çankırı, Turkey. \\ ${ }^{b}$ Eskişehir Osmangazi University, Department of Computer and Mathematics, Eskişehir, Turkey. \\ ${ }^{c}$ Department of Mathematics, Rasht Branch, Islamic Azad University, Rasht, Iran. \\ ${ }^{d}$ Faculty of Engineering Technology, Amol University of Special Modern Technologies, Amol, Iran. \\ ${ }^{e}$ Department of Mathematics, Faculty of Mathematical Sciences, University of Mazandaran, Babolsar, Iran.
}

\section{December 22, 2017}

\begin{abstract}
The Sine-Gordon expansion method is implemented to construct exact solutions some conformable time fractional equations in Regularized Long Wave(RLW)-class. Compatible wave transform reduces the governing equation to classical ordinary differential equation. The homogeneous balance procedure gives the order of the predicted polynomial-type solution that is inspired from well-known Sine-Gordon equation. The substitution of this solution follows the previous step. Equating the coefficients of the powers of predicted solution leads a system of algebraic equations. The solution of resultant system for coefficients gives the necessary relations among the parameters and the coefficients to be able construct the solutions. Some solutions are simulated for some particular choices of parameters.
\end{abstract}

Keywords: Sine-Gordon Expansion Method; Conformable time fractional RLW Equation; Conformable time fractional modified RLW Equation; Conformable time fractional symmetric-RLW Equation.

MSC2010: 35C07;35R11;35Q53.

PACS: 02.30.Jr; 02.70.Wz; 04.20.Jb

*alperkorkmaz7@gmail.com 


\section{Introduction}

Even though some nonlinear partial differential equations are integrable, it may be not easy to integrate them. Instead, a predicted solution with parameters are assumed to be a solution of governing equations and the relations among the parameters are investigated. The logic is simply based on the similarity with exponential-type solutions to the ordinary differential equations with constant coefficients. These predicted solutions are of various forms covering exponential, hyperbolic, trigonometric or rational functions, and more. Parallel to the recent developments in computer algebra in the last four decades, a tendency has been observed to determine exact solutions to nonlinear PDEs by following the procedure that starts with a predicted solution. Recently, this tendency has focused on exact solutions to fractional nonlinear partial differential equations. Many of techniques implemented to nonlinear PDEs to find exact solutions have been adapted for fractional nonlinear PDES [1-10]. We also derive exact solutions to some conformable fractional equations in RLW-class modeling various wave phenomena both in nature or technology implementations. Different from previous studies, we adapt Sine-Gordon expansion approach to determine exact solutions to governing equations in fractional RLW-class.

The first equation considered in this study is the RLW equation

$$
D_{t}^{\alpha} u+p u_{x}+q u u_{x}+r D_{t}^{\alpha} u_{x x}=0, \quad t \geq 0
$$

where $p, q$ and $r$ real coefficients, $D_{t}^{\alpha}$ conformable fractional differential operator and $u=u(x, t)$. The integer ordered form of the RLW equation describes formation and development of undular bore by a long wave in shallow water [11]. The same study investigates the transition and interaction between still water and a uniform flow. The origins and similarities to the KdV Equation of the RLW equation are discussed in general terms [12]. Moreover, Benjamin et al. developed an exact theory to the RLW equation and existence of classical solutions were proved. The Lagrangian density for the RLW equation was defined in [13]. Significant concept covering peak positions, amplitude and widths of two solitary waves were studied by trial function approach in the same paper.

The second equation to discuss exact solutions here is the modified RLW (mRLW) equation of the form

$$
D_{t}^{\alpha} u+p u_{x}+q u^{2} u_{x}+r D_{t}^{\alpha} u_{x x}=0, \quad t \geq 0
$$

where $D_{t}^{\alpha}$ conformable fractional differential operator. This equation seemed in Gardners' study [14]. B-spline finite elements based approximate solutions defining motion of solitary waves were investigated in that study. In the following decades, various numerical and exact solutions to different problems constructed on the mRLW equation are solved using diverse techniques [15-20]. 
The last equation to derive the exact solutions is the symmetric RLW (sRLW) equation

$$
D_{t t}^{2 \alpha} u+p u_{x x}+q u D_{t}^{\alpha} u_{x}+q u_{x} D_{t}^{\alpha} u+r D_{t t}^{2 \alpha} u_{x x}=0, \quad t \geq 0
$$

where $u=u(x, t), p, q$ and $r$ real parameters, $D_{t t}^{2 \alpha}$ is the fractional differential operator of order $2 \alpha$ in conformable sense. This equation is an interesting model to describe ion-acoustic and space charge waves with weak non linearity [21]. Before starting the solution procedure, we should give some significant properties of conformable fractional derivative. Thus, the next section focuses on conformable fractional derivative definition and some important properties. We explain the solution procedure in the third section. The following sections cover implementations of the proposed procedure to some conformable fractional PDEs in RLW-class.

\section{Conformable Fractional Derivative}

The conformable derivative of order $\alpha$ with respect to the independent variable $t$ is defined as

$$
D_{t}^{\alpha}(y(t))=\lim _{\tau \rightarrow 0} \frac{y\left(t+\tau t^{1-\alpha}\right)-y(t)}{\tau}, t>0, \alpha \in(0,1] .
$$

for a function $y=y(t):[0, \infty) \rightarrow \mathbb{R}[22]$. This newly defined fractional derivative is capable of satisfying some well known required properties.

Theorem 1 Assume that the order of the derivative $\alpha \in(0,1]$, and suppose that $u=u(t)$ and $y=y(t)$ are $\alpha$-differentiable for all positive $t$. Then,

- $D_{t}^{\alpha}\left(c_{1} u+c_{2} y\right)=c_{1} D_{t}^{\alpha}(u)+c_{2} D_{t}^{\alpha}(y)$

- $D_{t}^{\alpha}\left(t^{k}\right)=k t^{k-\alpha}, \forall k \in \mathbb{R}$

- $D_{t}^{\alpha}(\lambda)=0$, for all constant function $u(t)=\lambda$

- $D_{t}^{\alpha}(u y)=u D_{t}^{\alpha}(y)+y D_{t}^{\alpha}(u)$

- $D_{t}^{\alpha}\left(\frac{u}{y}\right)=\frac{y D_{t}^{\alpha}(u)-u D_{t}^{\alpha}(y)}{y^{2}}$

- $D_{t}^{\alpha}(u)(t)=t^{1-\alpha} \frac{d u}{d t}$

for $\forall c_{1}, c_{2} \in \mathbb{R}[23,24]$.

Conformable fractional differential operator satisfies some critical fundamental properties like the chain rule, Taylor series expansion and Laplace transform [25]. 
Theorem 2 Let $u=u(t)$ be an $\alpha$-conformable differentiable function and assume that $y$ is differentiable and defined in the range of $u$. Then,

$$
D_{t}^{\alpha}(u \circ y)(t)=t^{1-\alpha} y^{\prime}(t) u^{\prime}(y(t))
$$

\section{Sine-Gordon Expansion Method}

The classical wave transform $u(x, t)=U(\xi)$ with $\xi=a\left(x-\nu t^{\alpha} / \alpha\right)$ reduces the Sine-Gordon equation in one dimension of the form

$$
\frac{\partial^{2} u}{\partial x^{2}}-D_{t}^{2 \alpha} u=m^{2} \sin u, \quad m \text { is constant }
$$

to the ODE

$$
\frac{d^{2} U}{d \xi^{2}}=\frac{m^{2}}{a^{2}\left(1-\nu^{2}\right)} \sin U
$$

where $\nu$ represents velocity of the traveling wave defined in the transform [26]. Some simplifications lead

$$
\left(\frac{d(U / 2)}{d \xi}\right)^{2}=\frac{m^{2}}{a^{2}\left(1-\nu^{2}\right)} \sin ^{2} U / 2+C
$$

where $C$ is constant of integration. $C$ is assumed zero for simplicity. Let $w(\xi)=$ $U(\xi) / 2$ and $b^{2}=m^{2} /\left(a^{2}\left(1-\nu^{2}\right)\right)$. Then, (8) is converted to

$$
\frac{d(w)}{d \xi}=b \sin w
$$

Set $b=1$ in (9). Then, (9) yields two significant relations

$$
\sin w(\xi)=\left.\frac{2 d e^{\xi}}{d^{2} e^{2 \xi}+1}\right|_{d=1}=\operatorname{sech} \xi
$$

or

$$
\cos w(\xi)=\left.\frac{d^{2} e^{2 \xi}-1}{d^{2} e^{2 \xi}+1}\right|_{d=1}=\tanh \xi
$$

where $d$ is nonzero integral constant. The fractional PDE of the form

$$
P\left(u, D_{t}^{\alpha} u, u_{x}, D_{t t}^{2 \alpha} u, u_{x x}, \ldots\right)=0
$$

can be reduced to an ODE

$$
\tilde{P}\left(U, U^{\prime}, U^{\prime \prime}, \ldots\right)=0
$$


by using a compatible wave transform $u(x, t)=U(\xi)$ where the transform variable $\xi$ is defined as $a\left(x-\nu t^{\alpha} / \alpha\right)$. Then, the predicted solution to (13) of the form

$$
U(\xi)=A_{0}+\sum_{i=1}^{s} \tanh ^{i-1}(\xi)\left(B_{i} \operatorname{sech} \xi+A_{i} \tanh \xi\right)
$$

can be written as

$$
U(w)=A_{0}+\sum_{i=1}^{s} \cos ^{i-1}(w)\left(B_{i} \sin w+A_{i} \cos w\right)
$$

owing to $(10)$ - (11). The procedure starts by determining index limit $s$ by the assistance of homogenous balance of the terms in (13). Following the substitution of the predicted solution (15) into (13) the coefficients of powers of $\sin w \cos w$ are assumed as zero. Next, the resultant algebraic system is tried to be solved for the coefficients $A_{0}, A_{1}, B_{1}, \ldots, a, \nu$. Then, the solutions are constructed, if exists, by using (10) - (11) and $\xi$

\section{Solutions to the conformable time fractional RLW equation}

The traveling wave transform $u(x, t) \rightarrow U(\xi), \xi=a\left(x-\nu t^{\alpha} / \alpha\right)$ reduces the time fractional RLW equation to

$$
(-a \nu+a p) U+1 / 2 q a U^{2}-r \nu a^{3} U^{\prime \prime}=C
$$

where $C$ is constant of integration, and "denotes differentiation wrt $\xi$. Here, we assume that $C=0$ to reduce the complexity of the solutions. The balance between $U^{2}$ and $U^{\prime \prime}$ gives $s=2$. Thus, the predicted solution takes the form

$$
U(w)=A_{0}+B_{1} \sin w+A_{1} \cos w+B_{2} \cos w \sin w+A_{2} \cos ^{2} w
$$


Substituting this solution into (16) yields

$$
\begin{aligned}
& \left(-a^{3} \nu r B_{2}+a q A_{2} B_{2}\right) \sin (w(\xi))(\cos (w(\xi)))^{3} \\
& +(\cos (w(\xi)))^{3} a q A_{1} A_{2}+\left(a p A_{2}-a \nu A_{2}+1 / 2 a q A_{1}^{2}+a q A_{0} A_{2}\right)(\cos (w(\xi)))^{2} \\
& +\left(-a^{3} \nu r B_{1}+a q A_{1} B_{2}+a q A_{2} B_{1}\right) \sin (w(\xi))(\cos (w(\xi)))^{2} \\
& +\left(1 / 2 a q B_{2}^{2}+4 a^{3} \nu r A_{2}-1 / 2 a q A_{2}^{2}\right)(\sin (w(\xi)))^{2}(\cos (w(\xi)))^{2} \\
& +5(\sin (w(\xi)))^{3} \cos (w(\xi)) a^{3} \nu r B_{2} \\
& +\left(2 a^{3} \nu r A_{1}+a q B_{1} B_{2}\right)(\sin (w(\xi)))^{2} \cos (w(\xi)) \\
& +\left(a q A_{0} B_{2}+a q A_{1} B_{1}-a \nu B_{2}+a p B_{2}\right) \sin (w(\xi)) \cos (w(\xi)) \\
& +\left(a q A_{0} A_{1}-a \nu A_{1}+a p A_{1}\right) \cos (w(\xi)) \\
& +1 / 2 a q A_{2}^{2}+1 / 2 a q A_{0}^{2}-a \nu A_{0}+a p A_{0}+\left(a q A_{0} B_{1}-a \nu B_{1}+a p B_{1}\right) \sin (w(\xi)) \\
& +(\sin (w(\xi)))^{3} a^{3} \nu r B_{1}-2(\sin (w(\xi)))^{4} a^{3} \nu r A_{2} \\
& +\left(1 / 2 a q B_{1}^{2}-1 / 2 a q A_{2}^{2}\right)(\sin (w(\xi)))^{2}=0
\end{aligned}
$$

Using some trigonometric identities and simplifications we find the following algebraic system of equations:

$$
\begin{aligned}
1 / 2 a\left(-4 a^{2} \nu r A_{2}-q{A_{2}}^{2}+q B_{1}^{2}\right)+1 / 2 a q A_{2}{ }^{2}+1 / 2 a q A_{0}{ }^{2}-a \nu A_{0}+a p A_{0} & =0 \\
a^{3} \nu r B_{1}+a q A_{0} B_{1}-a \nu B_{1}+a p B_{1} & =0 \\
-a \nu A_{2}+a p A_{2}+1 / 2 a q A_{1}{ }^{2} & \\
+a q A_{0} A_{2}+6 a^{3} \nu r A_{2}+1 / 2 a q B_{2}{ }^{2}-1 / 2 a q A_{2}{ }^{2}-1 / 2 a\left(-4 a^{2} \nu r A_{2}-q A_{2}{ }^{2}+q B_{1}{ }^{2}\right) & =0 \\
-6 a^{3} \nu r A_{2}-1 / 2 a q B_{2}{ }^{2}+1 / 2 a q A_{2}{ }^{2} & =0 \\
-2 a^{3} \nu r A_{1}+a q A_{1} A_{2}-a q B_{1} B_{2} & =0 \\
2 a^{3} \nu r A_{1}+a q A_{0} A_{1}+a q B_{1} B_{2}-a \nu A_{1}+a p A_{1} & =0 \\
-6 a^{3} \nu r B_{2}+a q A_{2} B_{2} & =0 \\
-2 a^{3} \nu r B_{1}+a q A_{1} B_{2}+a q A_{2} B_{1} & =0 \\
5 a^{3} \nu r B_{2}+a q A_{0} B_{2}+a q A_{1} B_{1}-a \nu B_{2}+a p B_{2} & =0
\end{aligned}
$$

Solution of this system for $a, \nu, A_{0}, A_{1}, A_{2}, B_{1}$ and $B_{2}$ gives various solution sets with $a \neq 0$ and $\nu \neq 0$.

Set 1:

$$
\nu=\frac{p}{r a^{2}+1}, A_{0}=-\frac{6 p a^{2} r}{\left(r a^{2}+1\right) q}, A_{1}=0, A_{2} \quad=\frac{6 p a^{2} r}{\left(r a^{2}+1\right) q}, B_{1}=0, B_{2} \quad=\frac{6 r a^{2} p i}{\left(r a^{2}+1\right) q}, i=\sqrt{-1}
$$

Set 2:

$$
\nu=\frac{p}{r a^{2}+1}, A_{0}=-\frac{6 p a^{2} r}{\left(r a^{2}+1\right) q}, A_{1}=0, A_{2} \quad=\frac{6 p a^{2} r}{\left(r a^{2}+1\right) q}, B_{1}=0, B_{2} \quad=\frac{-6 r a^{2} p i}{\left(r a^{2}+1\right) q}, i=\sqrt{-1}
$$


Set 3:

$$
\nu=-\frac{p}{r a^{2}-1}, A_{0}=\frac{4 p a^{2} r}{\left(r a^{2}-1\right) q}, A_{1}=0, A_{2}=-\frac{6 p a^{2} r}{\left(r a^{2}-1\right) q}, B_{1}=0, B_{2} \quad=\frac{6 r a^{2} p i}{\left(r a^{2}-1\right) q}, i=\sqrt{-1}
$$

Set 4 :

$$
\nu=-\frac{p}{r a^{2}-1}, A_{0}=\frac{4 p a^{2} r}{\left(r a^{2}-1\right) q}, A_{1}=0, A_{2}=-\frac{6 p a^{2} r}{\left(r a^{2}-1\right) q}, B_{1}=0, B_{2} \quad=\frac{-6 r a^{2} p i}{\left(r a^{2}-1\right) q}, i=\sqrt{-1}
$$

Set 5:

$$
\nu=\frac{p}{4 r a^{2}+1}, A_{0}=-\frac{\frac{\left(8 r a^{2}-1\right) p}{4 r a^{2}+1}+p}{q}, A_{1}=0, A_{2}=\frac{12 p a^{2} r}{\left(4 r a^{2}+1\right) q}, B_{1}=0, B_{2}=0
$$

Set 6 :

$$
\nu=-\frac{p}{4 r a^{2}-1}, A_{0}=-\frac{-\frac{\left(8 r a^{2}-1\right) p}{4 r a^{2}-1}+p}{q}, A_{1}=0, A_{2}=-\frac{12 p a^{2} r}{\left(4 r a^{2}-1\right) q}, B_{1}=0, B_{2}=0
$$

Using these solution sets of algebraic equations, we construct the solutions to (1) as

$$
\begin{aligned}
& u_{1}(x, t)=-\frac{6 a^{2} r \nu}{q}+\frac{6 a^{2} r \nu}{q} i \tanh \left(a\left(x-\nu \frac{t^{\alpha}}{\alpha}\right)\right) \operatorname{sech}\left(a\left(x-\nu \frac{t^{\alpha}}{\alpha}\right)\right)+\frac{6 a^{2} r \nu}{q} \tanh ^{2}\left(a\left(x-\nu \frac{t^{\alpha}}{\alpha}\right)\right) \\
& u_{2}(x, t)=-\frac{6 a^{2} r \nu}{q}+\frac{6 a^{2} r \nu}{q} i \tanh \left(a\left(x-\nu \frac{t^{\alpha}}{\alpha}\right)\right) \operatorname{sech}\left(a\left(x-\nu \frac{t^{\alpha}}{\alpha}\right)\right)-\frac{6 a^{2} r \nu}{q} \tanh ^{2}\left(a\left(x-\nu \frac{t^{\alpha}}{\alpha}\right)\right)
\end{aligned}
$$

for $\nu=p /\left(r a^{2}+1\right)$,

$$
\begin{aligned}
& u_{3}(x, t)=-\frac{4 a^{2} r \nu}{q}+\frac{6 a^{2} r \nu}{q} i \tanh \left(a\left(x-\nu \frac{t^{\alpha}}{\alpha}\right)\right) \operatorname{sech}\left(a\left(x-\nu \frac{t^{\alpha}}{\alpha}\right)\right)-\frac{6 a^{2} r \nu}{q} \tanh ^{2}\left(a\left(x-\nu \frac{t^{\alpha}}{\alpha}\right)\right) \\
& u_{4}(x, t)=-\frac{4 a^{2} r \nu}{q}+\frac{6 a^{2} r \nu}{q} i \tanh \left(a\left(x-\nu \frac{t^{\alpha}}{\alpha}\right)\right) \operatorname{sech}\left(a\left(x-\nu \frac{t^{\alpha}}{\alpha}\right)\right)+\frac{6 a^{2} r \nu}{q} \tanh ^{2}\left(a\left(x-\nu \frac{t^{\alpha}}{\alpha}\right)\right)
\end{aligned}
$$

for $\nu=-p /\left(r a^{2}-1\right)$,

$$
u_{5}(x, t)=\frac{\left(8 r a^{2}-1\right) \nu+p}{q}+\frac{12 a^{2} r \nu}{q} \tanh ^{2}\left(a\left(x-\nu \frac{t^{\alpha}}{\alpha}\right)\right)
$$

for $\nu=p /\left(4 r a^{2}+1\right)$.

$$
u_{6}(x, t)=-\frac{\left(8 r a^{2}-1\right) \nu+p}{q}+\frac{12 a^{2} r \nu}{q} \tanh ^{2}\left(a\left(x-\nu \frac{t^{\alpha}}{\alpha}\right)\right)
$$

for $\nu=-p /\left(4 r a^{2}+1\right)$.

A particular form of $u_{5}(x, t)$ is depicted for various values of $\alpha \in\{0.25,0.5,0.75,1\}$ in a finite domain in Fig $1(\mathrm{a})-1(\mathrm{~d})$. It is observed that the propagation of the initial pulse propagates along the $x$-axis as time proceeds by preserving its shape and amplitude in all cases. $\alpha$ affects only propagation velocity. The propagation is faster in small times but later it gets slower when $\alpha$ is less than $1 . \alpha=1$ choice gives a constant propagation velocity to the pulse, Fig $1(\mathrm{~d})$. 


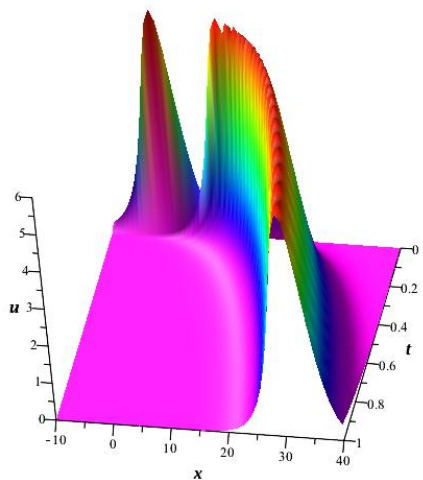

(a) $\alpha=0.25$

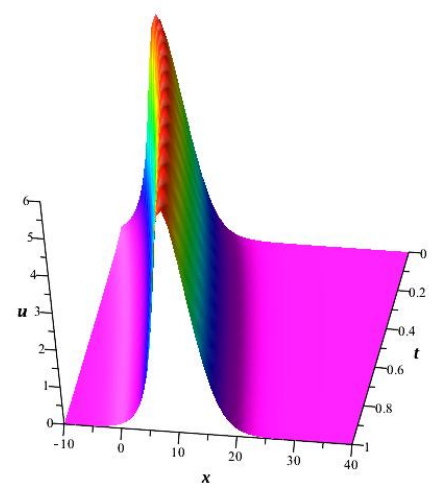

(c) $\alpha=0.75$

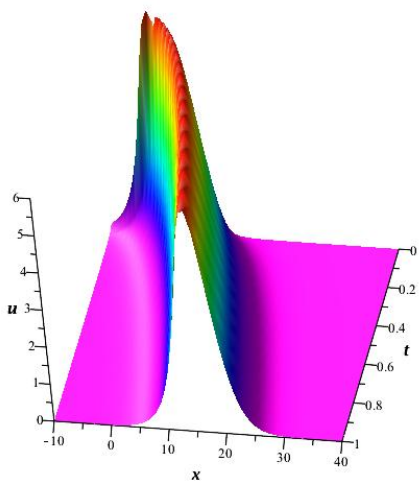

(b) $\alpha=0.50$

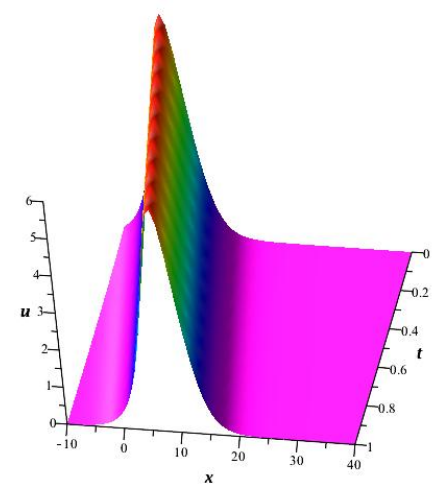

(d) $\alpha=1$

Figure 1: The solution $u_{5}(x, t)$ for $\{a=1 / 4, q=-1, r=1, p=10\}$

\section{Solutions to the conformable time fractional mRLW equation}

The traveling wave transform $u(x, t) \rightarrow U(\xi), \xi=a\left(x-\nu t^{\alpha} / \alpha\right)$ reduces the mRLW equation (2) to

$$
(-a \nu+a p) U+\frac{q a}{3} U^{3}-r \nu a^{3} U^{\prime \prime}=C
$$

where $C$ is constant of integration. The balance between $U^{3}$ and $U^{\prime \prime}$ leads $s=1$. Thus, the predicted solution takes the form

$$
U(w)=A_{0}+A_{1} \cos w+B_{1} \sin w
$$

Substituting the predicted solution (27) into (26) gives 


$$
\begin{aligned}
& 1 / 3(\cos (w(\xi)))^{3} a q A_{1}{ }^{3} \\
& +\left(-a^{3} \nu r B_{1}+a q A_{1}{ }^{2} B_{1}\right) \sin (w(\xi))(\cos (w(\xi)))^{2} \\
& +(\cos (w(\xi)))^{2} a q A_{0} A_{1}{ }^{2}+\left(2 a^{3} \nu r A_{1}+a q A_{1} B_{1}{ }^{2}\right)(\sin (w(\xi)))^{2} \cos (w(\xi)) \\
& +2 \sin (w(\xi)) \cos (w(\xi)) a q A_{0} A_{1} B_{1}+\left(a q A_{0}{ }^{2} A_{1}-a \nu A_{1}+a p A_{1}\right) \cos (w(\xi)) \\
& -C-a \nu A_{0}+a p A_{0}+1 / 3 a q A_{0}{ }^{3}+\left(a^{3} \nu r B_{1}+1 / 3 a q B_{1}{ }^{3}\right)(\sin (w(\xi)))^{3} \\
& +(\sin (w(\xi)))^{2} a q A_{0} B_{1}{ }^{2} \\
& +\left(a q A_{0}{ }^{2} B_{1}-a \nu B_{1}+a p B_{1}\right) \sin (w(\xi))=0
\end{aligned}
$$

Following implementation of some trigonometric identities and some simplifications, we equate the coefficients of powers of sin, cos and multiplications of them to zero to give the algebraic system of equations

$$
\begin{aligned}
a q A_{0} B_{1}{ }^{2}-C-a \nu A_{0}+a p A_{0}+1 / 3 a q A_{0}{ }^{3} & =0 \\
a q A_{0}{ }^{2} B_{1}-a \nu B_{1}+a p B_{1}+a^{3} \nu r B_{1}+1 / 3 a q B_{1}{ }^{3} & =0 \\
a q A_{0} A_{1}{ }^{2}-a q A_{0} B_{1}{ }^{2} & =0 \\
-2 a^{3} \nu r B_{1}+a q A_{1}{ }^{2} B_{1}-1 / 3 a q B_{1}{ }^{3} & =0 \\
-2 a^{3} \nu r A_{1}-a q A_{1} B_{1}{ }^{2}+1 / 3 a q A_{1}^{3} & =0 \\
2 a q A_{0} A_{1} B_{1} & =0 \\
2 a^{3} \nu r A_{1}+a q A_{0}{ }^{2} A_{1}+a q A_{1} B_{1}{ }^{2}-a \nu A_{1}+a p A_{1} & =0
\end{aligned}
$$


The solution of this system for $\nu, A_{0}, A_{1}, B_{1}$ and $C$ forces $C$ to be zero. Thus, the solutions of this system can be summarized as

$$
\begin{array}{rlrlrl}
\nu & =-\frac{-2 p q}{q r a^{2}-2 q}, & A_{0} & =0, A_{1}=\sqrt{\frac{3 r \nu}{2 q} a,} & B_{1}=\sqrt{\frac{-3 r \nu}{2 q}} a \\
\nu & =-\frac{-2 p q}{q r a^{2}-2 q}, & A_{0} & =0, A_{1}=\sqrt{\frac{3 r \nu}{2 q} a,} & B_{1}=-\sqrt{\frac{-3 r \nu}{2 q}} a \\
\nu & =-\frac{-2 p q}{q r a^{2}-2 q}, & A_{0} & =0, A_{1}=-\sqrt{\frac{3 r \nu}{2 q}} a, & B_{1}=\sqrt{\frac{-3 r \nu}{2 q}} a \\
\nu & =-\frac{-2 p q}{q r a^{2}-2 q}, & A_{0} & =0, A_{1}=-\sqrt{\frac{3 r \nu}{2 q}} a, & B_{1}=-\sqrt{\frac{-3 r \nu}{2 q}} a \\
\nu=\frac{p}{r a^{2}+1}, & A_{0}=0, A_{1}=0, & B_{1}=\sqrt{\frac{6 r \nu}{q}} a \\
\nu=\frac{p}{r a^{2}+1}, & A_{0}=0, A_{1}=0, & B_{1}=-\sqrt{\frac{6 r \nu}{q}} a \\
\nu=-\frac{p}{2 r a^{2}-1}, & A_{0}=0, A_{1}=\sqrt{\frac{-6 r \nu}{q}} a, & & B_{1}=0 \\
\nu & =-\frac{p}{2 r a^{2}-1}, & A_{0} & =0, A_{1}=-\sqrt{\frac{-6 r \nu}{q}} a, & & B_{1}=0
\end{array}
$$


for arbitrary $a$. When $a$ is considered as non arbitrary, we have some more solutions such that

$$
\begin{array}{lll}
a & =\sqrt{\frac{1}{r}}, \quad \nu=2 p, A_{0}=0, & A_{1}=\sqrt{\frac{3 p}{q}}, B_{1}=\sqrt{\frac{-3 p}{q}} \\
a & =-\sqrt{\frac{1}{r}}, \quad \nu=2 p, A_{0}=0, & A_{1}=\sqrt{\frac{3 p}{q}}, B_{1}=\sqrt{\frac{-3 p}{q}} \\
a & =\sqrt{\frac{1}{r}}, \quad \nu=2 p, A_{0}=0, & A_{1}=-\sqrt{\frac{3 p}{q}}, B_{1}=\sqrt{\frac{-3 p}{q}} \\
a & =-\sqrt{\frac{1}{r}}, \quad \nu=2 p, A_{0}=0, & A_{1}=-\sqrt{\frac{3 p}{q}}, B_{1}=\sqrt{\frac{-3 p}{q}} \\
a & =\sqrt{\frac{1}{r}}, \quad \nu=2 p, A_{0}=0, & A_{1}=\sqrt{\frac{3 p}{q}}, B_{1}=-\sqrt{\frac{-3 p}{q}} \\
a & =-\sqrt{\frac{1}{r}}, \quad \nu=2 p, A_{0}=0, & A_{1}=\sqrt{\frac{3 p}{q}}, B_{1}=-\sqrt{\frac{-3 p}{q}} \\
a & =\sqrt{\frac{1}{r}}, \quad \nu=2 p, A_{0}=0, & A_{1}=-\sqrt{\frac{3 p}{q}}, B_{1}=-\sqrt{\frac{-3 p}{q}} \\
a & =-\sqrt{\frac{1}{r}}, \quad \nu=2 p, A_{0}=0, & A_{1}=-\sqrt{\frac{3 p}{q}}, B_{1}=-\sqrt{\frac{-3 p}{q}}
\end{array}
$$


Thus, the solutions to mRLW equation (2) are represented by

$$
\begin{aligned}
& u_{7}(x, t)=\sqrt{\frac{3 r \nu}{2 q}} a \tanh \left(a\left(x-\nu \frac{t^{\alpha}}{\alpha}\right)\right)+\sqrt{\frac{-3 r \nu}{2 q}} a \operatorname{sech}\left(a\left(x-\nu \frac{t^{\alpha}}{\alpha}\right)\right), \nu=-\frac{-2 p q}{q r a^{2}-2 q} \\
& u_{8}(x, t)=\sqrt{\frac{3 r \nu}{2 q}} a \tanh \left(a\left(x-\nu \frac{t^{\alpha}}{\alpha}\right)\right)-\sqrt{\frac{-3 r \nu}{2 q}} a \operatorname{sech}\left(a\left(x-\nu \frac{t^{\alpha}}{\alpha}\right)\right), \nu=-\frac{-2 p q}{q r a^{2}-2 q} \\
& u_{9}(x, t)=-\sqrt{\frac{3 r \nu}{2 q}} a \tanh \left(a\left(x-\nu \frac{t^{\alpha}}{\alpha}\right)\right)+\sqrt{\frac{-3 r \nu}{2 q}} a \operatorname{sech}\left(a\left(x-\nu \frac{t^{\alpha}}{\alpha}\right)\right), \nu=-\frac{-2 p q}{q r a^{2}-2 q} \\
& u_{10}(x, t)=-\sqrt{\frac{3 r \nu}{2 q}} a \tanh \left(a\left(x-\nu \frac{t^{\alpha}}{\alpha}\right)\right)-\sqrt{\frac{-3 r \nu}{2 q}} a \operatorname{sech}\left(a\left(x-\nu \frac{t^{\alpha}}{\alpha}\right)\right), \nu=-\frac{-2 p q}{q r a^{2}-2 q} \\
& u_{11}(x, t)=\sqrt{\frac{6 r \nu}{q}} a \operatorname{sech}\left(a\left(x-\nu \frac{t^{\alpha}}{\alpha}\right)\right), \nu=\frac{p}{r a^{2}+1} \\
& u_{12}(x, t)=-\sqrt{\frac{6 r \nu}{q}} a \operatorname{sech}\left(a\left(x-\nu \frac{t^{\alpha}}{\alpha}\right)\right), \nu=\frac{p}{r a^{2}+1} \\
& u_{13}(x, t)=\sqrt{\frac{-6 r \nu}{q}} a \tanh \left(a\left(x-\nu \frac{t^{\alpha}}{\alpha}\right)\right), \nu=-\frac{-p}{2 r a^{2}-1} \\
& u_{14}(x, t)=-\sqrt{\frac{-6 r \nu}{q}} a \tanh \left(a\left(x-\nu \frac{t^{\alpha}}{\alpha}\right)\right), \nu=-\frac{-p}{2 r a^{2}-1}
\end{aligned}
$$


when $\nu$ is dependent on $a$. Whenever $\nu$ and $a$ are independent upon each other, the solution are represented by

$$
\begin{aligned}
& u_{15}(x, t)=\sqrt{\frac{3 p}{q}} \tanh \left(a\left(x-\nu \frac{t^{\alpha}}{\alpha}\right)\right)+\sqrt{\frac{-3 p}{q}} \operatorname{sech}\left(a\left(x-\nu \frac{t^{\alpha}}{\alpha}\right)\right), \nu=2 p, a=\sqrt{\frac{1}{r}} \\
& u_{16}(x, t)=\sqrt{\frac{3 p}{q}} \tanh \left(a\left(x-\nu \frac{t^{\alpha}}{\alpha}\right)\right)+\sqrt{\frac{-3 p}{q}} \operatorname{sech}\left(a\left(x-\nu \frac{t^{\alpha}}{\alpha}\right)\right), \nu=2 p, a=-\sqrt{\frac{1}{r}} \\
& u_{17}(x, t)=-\sqrt{\frac{3 p}{q}} \tanh \left(a\left(x-\nu \frac{t^{\alpha}}{\alpha}\right)\right)+\sqrt{\frac{-3 p}{q}} \operatorname{sech}\left(a\left(x-\nu \frac{t^{\alpha}}{\alpha}\right)\right), \nu=2 p, a=\sqrt{\frac{1}{r}} \\
& u_{18}(x, t)=-\sqrt{\frac{3 p}{q}} \tanh \left(a\left(x-\nu \frac{t^{\alpha}}{\alpha}\right)\right)+\sqrt{\frac{-3 p}{q}} \operatorname{sech}\left(a\left(x-\nu \frac{t^{\alpha}}{\alpha}\right)\right), \nu=2 p, a=-\sqrt{\frac{1}{r}} \\
& u_{19}(x, t)=\sqrt{\frac{3 p}{q}} \tanh \left(a\left(x-\nu \frac{t^{\alpha}}{\alpha}\right)\right)-\sqrt{\frac{-3 p}{q}} \operatorname{sech}\left(a\left(x-\nu \frac{t^{\alpha}}{\alpha}\right)\right), \nu=2 p, a=\sqrt{\frac{1}{r}} \\
& u_{20}(x, t)=\sqrt{\frac{3 p}{q}} \tanh \left(a\left(x-\nu \frac{t^{\alpha}}{\alpha}\right)\right)-\sqrt{\frac{-3 p}{q}} \operatorname{sech}\left(a\left(x-\nu \frac{t^{\alpha}}{\alpha}\right)\right), \nu=2 p, a=-\sqrt{\frac{1}{r}} \\
& u_{21}(x, t)=-\sqrt{\frac{3 p}{q}} \tanh \left(a\left(x-\nu \frac{t^{\alpha}}{\alpha}\right)\right)-\sqrt{\frac{-3 p}{q}} \operatorname{sech}\left(a\left(x-\nu \frac{t^{\alpha}}{\alpha}\right)\right), \nu=2 p, a=\sqrt{\frac{1}{r}} \\
& u_{22}(x, t)=-\sqrt{\frac{3 p}{q}} \tanh \left(a\left(x-\nu \frac{t^{\alpha}}{\alpha}\right)\right)-\sqrt{\frac{-3 p}{q}} \operatorname{sech}\left(a\left(x-\nu \frac{t^{\alpha}}{\alpha}\right)\right), \nu=2 p, a=-\sqrt{\frac{1}{r}}
\end{aligned}
$$

The solution $u_{11}(x, t)$ is simulated for various values of $\alpha$ in Fig 2(a) - 2(d) by using the parameter set $\{a=1, q=-1, r=1 / 3, p=10\}$. The solution propagates along $x$-axis as time proceeds with preserved shape and amplitude. The propagation is not linear when $\alpha$ is less than 1, Fig 2(a)-2(c) as we observe a linear propagation with constant speed with $\alpha=1$, Fig 2(d). 


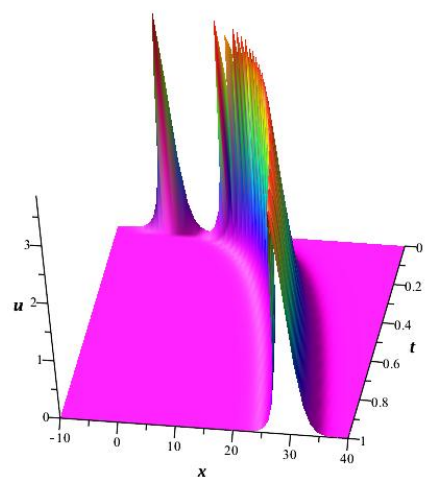

(a) $\alpha=0.25$

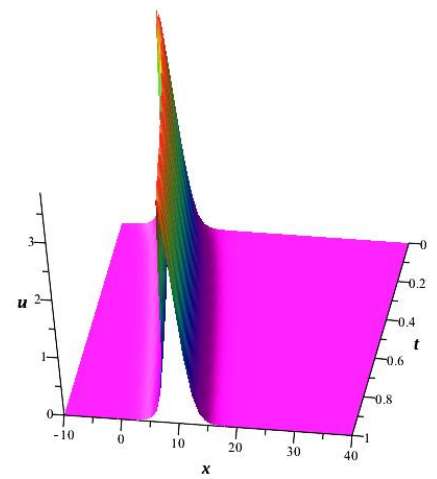

(c) $\alpha=0.75$

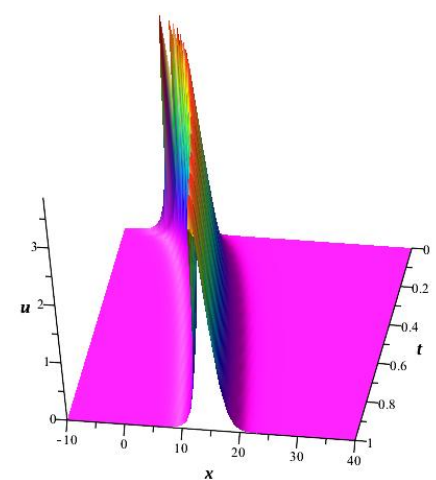

(b) $\alpha=0.50$

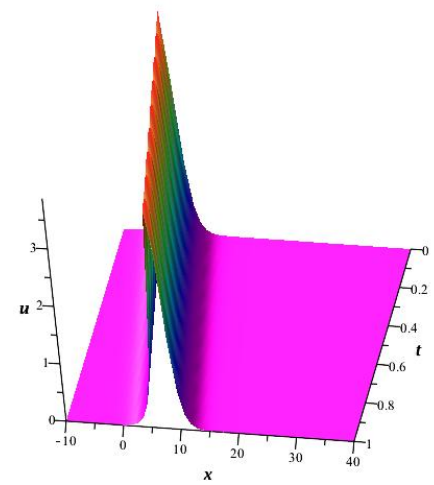

(d) $\alpha=1$

Figure 2: The solution $u_{11}(x, t)$ for $\{a=1, q=-1, r=1 / 3, p=10\}$

\section{Solutions to the conformable time fractional sRLW equation}

The traveling wave transform $u(x, t) \rightarrow U(\xi), \xi=a\left(x-\nu t^{\alpha} / \alpha\right)$ reduces the sRLW equation (2) to

$$
\left(a^{2} \nu^{2}+a^{2} p\right) U^{\prime}+r \nu^{2} a^{4} U^{\prime \prime \prime}-q \nu a^{2} U U^{\prime}=C
$$

The balance between $U^{\prime \prime \prime}$ and $U U^{\prime}$ gives $s=2$. Thus, we seek a solution of the form

$$
U(w)=A_{0}+A_{1} \cos w+B_{1} \sin w+B_{2} \cos w \sin w+A_{2} \cos ^{2} w
$$


Substitution of this solution into (34) gives

$$
\begin{aligned}
& a^{4} \nu^{2} r B_{2} \sin (w(\xi))(\cos (w(\xi)))^{4} \\
& +\left(-8 a^{4} \nu^{2} r A_{2}+2 a^{2} \nu q A_{2}^{2}-a^{2} \nu q B_{2}^{2}\right)(\sin (w(\xi)))^{2}(\cos (w(\xi)))^{3} \\
& +\left(a^{4} \nu^{2} r B_{1}-a^{2} \nu q A_{1} B_{2}-a^{2} \nu q A_{2} B_{1}\right) \sin (w(\xi))(\cos (w(\xi)))^{3} \\
& +\left(-18 a^{4} \nu^{2} r B_{2}+4 a^{2} \nu q A_{2} B_{2}\right)(\sin (w(\xi)))^{3}(\cos (w(\xi)))^{2} \\
& +\left(-4 a^{4} \nu^{2} r A_{1}+3 a^{2} \nu q A_{1} A_{2}-2 a^{2} \nu q B_{1} B_{2}\right)(\sin (w(\xi)))^{2}(\cos (w(\xi)))^{2} \\
& +\left(-a^{2} \nu q A_{0} B_{2}-a^{2} \nu q A_{1} B_{1}+a^{2} \nu^{2} B_{2}+a^{2} p B_{2}\right) \sin (w(\xi))(\cos (w(\xi)))^{2} \\
& +\left(16 a^{4} \nu^{2} r A_{2}+a^{2} \nu q B_{2}^{2}\right)(\sin (w(\xi)))^{4} \cos (w(\xi)) \\
& +\left(-5 a^{4} \nu^{2} r B_{1}+2 a^{2} \nu q A_{1} B_{2}+2 a^{2} \nu q A_{2} B_{1}\right)(\sin (w(\xi)))^{3} \cos (w(\xi)) \\
& +\left(2 a^{2} \nu q A_{0} A_{2}+a^{2} \nu q A_{1}^{2}-a^{2} \nu q B_{1}^{2}-2 a^{2} \nu^{2} A_{2}-2 a^{2} p A_{2}\right)(\sin (w(\xi)))^{2} \cos (w(\xi)) \\
& +\left(-a^{2} \nu q A_{0} B_{1}+a^{2} \nu^{2} B_{1}+a^{2} p B_{1}\right) \sin (w(\xi)) \cos (w(\xi))+5(\sin (w(\xi)))^{5} a^{4} \nu^{2} r B_{2} \\
& +\left(2 a^{4} \nu^{2} r A_{1}+a^{2} \nu q B_{1} B_{2}\right)(\sin (w(\xi)))^{4} \\
& +\left(a^{2} \nu q A_{0} B_{2}+a^{2} \nu q A_{1} B_{1}+a^{2} \nu q A_{2} B_{2}-a^{2} \nu^{2} B_{2}-a^{2} p B_{2}\right)(\sin (w(\xi)))^{3} \\
& +\left(a^{2} \nu q A_{0} A_{1}-a^{2} \nu^{2} A_{1}-a^{2} p A_{1}\right)(\sin (w(\xi)))^{2} \\
& -a^{2} \nu q A_{2} B_{2} \sin (w(\xi))-C=0
\end{aligned}
$$

Substitution of some trigonometric identities and some simplifications leads the following system of algebraic equations from the equating the coefficients of powers of cos, sin functions and their multiplications: 


$$
\begin{aligned}
-3 a^{2} \nu\left(2 a^{2} \nu r A_{1}-q A_{1} A_{2}+q B_{1} B_{2}\right)+8 a^{4} \nu^{2} r A_{1}+a^{2} \nu q A_{0} A_{1}-3 a^{2} \nu q A_{1} A_{2} & \\
+4 a^{2} \nu q B_{1} B_{2}-a^{2} \nu^{2} A_{1}-a^{2} p A_{1}-C & =0 \\
5 a^{4} \nu^{2} r B_{2}+a^{2} \nu q A_{0} B_{2}+a^{2} \nu q A_{1} B_{1}-a^{2} \nu^{2} B_{2}-a^{2} p B_{2} & =0 \\
24 a^{4} \nu^{2} r B_{2}-4 a^{2} \nu q A_{2} B_{2} & =0 \\
6 a^{4} \nu^{2} r B_{1}-3 a^{2} \nu q A_{1} B_{2}-3 a^{2} \nu q A_{2} B_{1} & =0 \\
-28 a^{4} \nu^{2} r B_{2}-2 a^{2} \nu q A_{0} B_{2}-2 a^{2} \nu q A_{1} B_{1} & \\
+3 a^{2} \nu q A_{2} B_{2}+2 a^{2} \nu^{2} B_{2}+2 a^{2} p B_{2} & =0 \\
-5 a^{4} \nu^{2} r B_{1}-a^{2} \nu q A_{0} B_{1}+2 a^{2} \nu q A_{1} B_{2} & \\
+2 a^{2} \nu q A_{2} B_{1}+a^{2} \nu^{2} B_{1}+a^{2} p B_{1} & =0 \\
a^{2}\left(24 a^{2} \nu^{2} r A_{2}-2 \nu q A_{2}{ }^{2}+2 \nu q B_{2}{ }^{2}\right) & =0 \\
6 a^{4} \nu^{2} r A_{1}-3 a^{2} \nu q A_{1} A_{2}+3 a^{2} \nu q B_{1} B_{2} & =0 \\
a^{2}\left(-16 a^{2} \nu^{2} r A_{2}-2 \nu q A_{0} A_{2}-\nu q A_{1}{ }^{2}+\nu q B_{1}{ }^{2}-\nu q B_{2}{ }^{2}+2 \nu^{2} A_{2}+2 p A_{2}\right) & \\
-a^{2}\left(24 a^{2} \nu^{2} r A_{2}-2 \nu q A_{2}{ }^{2}+2 \nu q B_{2}{ }^{2}\right) & =0 \\
-14 a^{4} \nu^{2} r A_{1}-a^{2} \nu q A_{0} A_{1}+6 a^{2} \nu q A_{1} A_{2}-7 a^{2} \nu q B_{1} B_{2} & \\
+a^{2} \nu^{2} A_{1}+a^{2} p A_{1}+3 a^{2} \nu\left(2 a^{2} \nu r A_{1}-q A_{1} A_{2}+q B_{1} B_{2}\right) & =0 \\
-a^{2}\left(-16 a^{2} \nu^{2} r A_{2}-2 \nu q A_{0} A_{2}-\nu q A_{1}{ }^{2}+\nu q B_{1}{ }^{2}-\nu q B_{2}{ }^{2}+2 \nu^{2} A_{2}+2 p A_{2}\right) & =0
\end{aligned}
$$

Solution of this system gives

$$
\begin{aligned}
& A_{0}=-\frac{5 a^{2} \nu^{2} r-\nu^{2}-p}{\nu q}, \quad A_{1}=0, A_{2}=\frac{12 a^{2} \nu r}{q}, \quad B_{1}=0, B_{2} \quad=0 \\
& A_{0}=-\frac{5 a^{2} \nu^{2} r-\nu^{2}-p}{\nu q}, \quad A_{1}=0, A_{2}=\frac{6 a^{2} \nu r}{q}, \quad B_{1}=0, B_{2}=\frac{6 a^{2} r \nu i}{q} \\
& A_{0}=-\frac{5 a^{2} \nu^{2} r-\nu^{2}-p}{\nu q}, \quad A_{1}=0, A_{2}=\frac{6 a^{2} \nu r}{q}, \quad B_{1}=0, B_{2}=-\frac{6 a^{2} r \nu i}{q}
\end{aligned}
$$

for arbitrarily chosen $\nu, a$ and $C=0$. One should note that the solution of this system is determinable whenever $C=0$. Thus, the solutions to (3) are constructed 
as

$$
\begin{aligned}
u_{23}(x, t) & =-\frac{5 a^{2} \nu^{2} r-\nu^{2}-p}{\nu q}+\frac{6 a^{2} r \nu}{q} i \tanh \left(a\left(x-\nu \frac{t^{\alpha}}{\alpha}\right)\right) \operatorname{sech}\left(a\left(x-\nu \frac{t^{\alpha}}{\alpha}\right)\right) \\
& +\frac{6 a^{2} r \nu}{q} \tanh ^{2}\left(a\left(x-\nu \frac{t^{\alpha}}{\alpha}\right)\right) \\
u_{24}(x, t) & =-\frac{5 a^{2} \nu^{2} r-\nu^{2}-p}{\nu q}+\frac{6 a^{2} r \nu}{q} i \tanh \left(a\left(x-\nu \frac{t^{\alpha}}{\alpha}\right)\right) \operatorname{sech}\left(a\left(x-\nu \frac{t^{\alpha}}{\alpha}\right)\right) \\
& -\frac{6 a^{2} r \nu}{q} \tanh ^{2}\left(a\left(x-\nu \frac{t^{\alpha}}{\alpha}\right)\right) \\
u_{25}(x, t) & =-\frac{8 a^{2} \nu^{2} r-\nu^{2}-p}{\nu q}+\frac{12 a^{2} \nu r}{q} \tanh ^{2}\left(a\left(x-\nu \frac{t^{\alpha}}{\alpha}\right)\right)
\end{aligned}
$$

The solution $u_{25}(x, t)$ is depicted in Fig 3(a)-3(d) for various $\alpha$ values in some finite domain of independent variables. The initial pulse propagates along the space axis without changing its shape and amplitude in all cases. $\alpha$ affects only propagation velocity due to being multiplier of time variable. Even though the pulse propagates non linearly for smaller $\alpha$ values, the propagation is linear when $\alpha$ is 1 . 


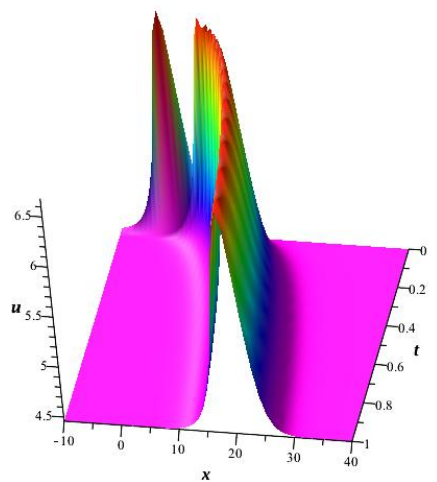

(a) $\alpha=0.25$

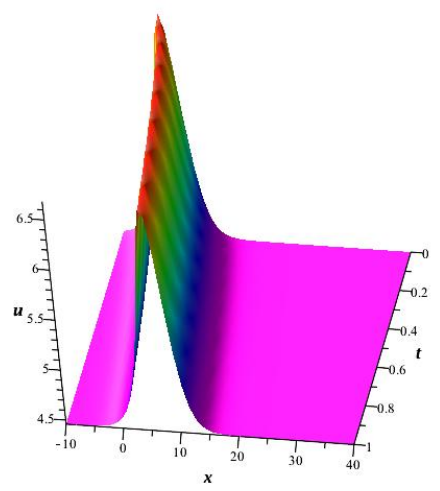

(c) $\alpha=0.75$

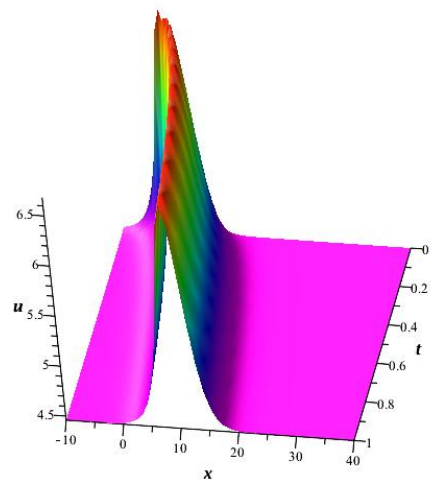

(b) $\alpha=0.50$

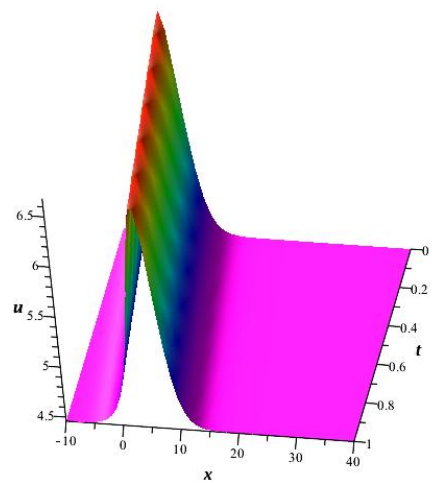

(d) $\alpha=1$

Figure 3: The solution $u_{25}(x, t)$ for $\{a=1, q=1, r=-1 / 3, p=1, \nu=5\}$

\section{Conclusion}

In the paper, exact solutions of some conformable fractional equations in the RLWclass are investigated by using Sine-Gordon expansion approach. Using compatible wave transform, the equations are reduced to some ODEs. Then, the predicted solutions are substituted into the resultant ODE. Equating the coefficients of cosine and sine functions and their multiplications to zero leads to some algebraic system of equation. Solving this system gives the relations among the parameters. In conclusion, some real and complex solutions that are combinations of powers of hyperbolic tangent and hyperbolic secant functions are determined explicitly. Graphical representations of some real valued solutions are depicted in some finite domains to comprehend the effects of $\alpha$. 


\section{References}

[1] Korkmaz, A., \& Hosseini, K. (2017). Exact solutions of a nonlinear conformable time-fractional parabolic equation with exponential nonlinearity using reliable methods. Optical and Quantum Electronics, 49(8), 278.

[2] Hosseini, K., \& Ansari, R. (2017). New exact solutions of nonlinear conformable time-fractional Boussinesq equations using the modified Kudryashov method. Waves in Random and Complex Media, 1-9.

[3] Korkmaz A., On The Wave Solutions of Conformable Fractional Evolution Equations, Commun. Fac. Sci. Univ. Ank. Series A1, 67(1) 68-79, 2018.

[4] Korkmaz A., Exact Solutions to $(3+1)$ Conformable Time Fractional Jimbo-Miwa,Zakharov-Kuznetsov and Modified Zakharov-Kuznetsov Equations, Communications in Theoretical Physics, 2017, 67(5), 479-482.

[5] Kaplan, M., \& Hosseini, K. (2018). Investigation of exact solutions for the Tzitzéica type equations in nonlinear optics. Optik-International Journal for Light and Electron Optics, 154, 393-397.

[6] Hosseini, K., Mayeli, P., \& Ansari, R. (2017). Bright and singular soliton solutions of the conformable time-fractional Klein-Gordon equations with different nonlinearities. Waves in Random and Complex Media, 1-9.

[7] Hosseini, K., Mayeli, P., \& Ansari, R. (2017). Modified Kudryashov method for solving the conformable time-fractional Klein-Gordon equations with quadratic and cubic nonlinearities. Optik-International Journal for Light and Electron Optics, 130, 737-742.

[8] Kaplan, M., Mayeli, P., \& Hosseini, K. (2017). Exact traveling wave solutions of the Wu-Zhang system describing $(1+1)$-dimensional dispersive long wave. Optical and Quantum Electronics, 49(12), 404.

[9] Tasbozan, O., Çenesiz, Y., \& Kurt, A. (2016). New solutions for conformable fractional Boussinesq and combined KdV-mKdV equations using Jacobi elliptic function expansion method. The European Physical Journal Plus, 131(7), 244.

[10] Çenesiz, Y., Tasbozan, O., \& Kurt, A. (2017). Functional Variable Method for conformable fractional modified KdV-ZK equation and Maccari system. Tbilisi Mathematical Journal, 10(1), 117-125.

[11] Peregrine, D. H. (1966). Calculations of the development of an undular bore. Journal of Fluid Mechanics, 25(02), 321-330. 
[12] Benjamin, T. B., Bona, J. L., \& Mahony, J. J. (1972). Model equations for long waves in nonlinear dispersive systems. Philosophical Transactions of the Royal Society of London A: Mathematical, Physical and Engineering Sciences, 272(1220), 47-78.

[13] Morrison, P. J., Meiss, J. D., \& Cary, J. R. (1984). Scattering of regularizedlong-wave solitary waves. Physica D: Nonlinear Phenomena, 11(3), 324-336.

[14] Gardner LRT, Gardner GA, Ayoub FA, \& Amein NK. (1997). Approximations of solitary waves of the MRLW equation by B-spline finite element. Arab Journal of Science Eng, 22,183-193.

[15] Soliman, A. M. A. (2017). Collocation Method using Quartic B-Splines for Solving the Modified RLW Equation. Indian Journal of Science and Technology, $10(31)$.

[16] Khalifa, A. K., Raslan, K. R., \& Alzubaidi, H. M. (2008). A collocation method with cubic B-splines for solving the MRLW equation. Journal of Computational and Applied Mathematics, 212(2), 406-418.

[17] Dereli, Y. (2012). Solitary wave solutions of the MRLW equation using radial basis functions. Numerical Methods for Partial Differential Equations, 28(1), 235-247.

[18] Karakoc, S. B. G., \& Geyikli, T. (2013). Petrov-Galerkin finite element method for solving the MRLW equation. Mathematical Sciences, 7(1), 25.

[19] Karakoc, S. B. G., Yagmurlu, N. M., \& Ucar, Y. (2013). Numerical approximation to a solution of the modified regularized long wave equation using quintic B-splines. Boundary Value Problems, 2013(1), 27.

[20] Keskin, P., \& Irk, D. (2012). Numerical solution of the MRLW equation using finite difference method. International Journal of Nonlinear Science, 14(3), 355-361.

[21] Seyler, C. E., \& Fenstermacher, D. L. (1984). A symmetric regularized-longwave equation. Physics of Fluids (1958-1988), 27(1), 4-7.

[22] Khalil, R., Al Horani, M., Yousef, A., \& Sababheh, M. (2014). A new definition of fractional derivative. Journal of Computational and Applied Mathematics, 264, 65-70.

[23] Atangana, A., Baleanu, D., \& Alsaedi, A. (2015). New properties of conformable derivative. Open Mathematics, 13(1), 1-10. 
[24] Çenesiz, Y., Baleanu, D., Kurt, A., \& Tasbozan, O. (2016). New exact solutions of Burgers' type equations with conformable derivative. Waves in Random and Complex Media, 1-14.

[25] Abdeljawad, T. (2015). On conformable fractional calculus. Journal of computational and Applied Mathematics, 279, 57-66.

[26] Yan, C. (1996), A simple transformation for nonlinear waves, Physics Letters A, 224, 77-84. 